\title{
Connectomes as constitutively epistemic objects: critical perspectives on modeling in current neuroanatomy
}

\author{
Philipp Haueis ${ }^{1,2}$ and Jan Slaby ${ }^{3}$ \\ ${ }^{1}$ Berlin School of Mind and Brain, Berlin, Germany \\ ${ }^{2}$ Research Group for Neuroanatomy and Connectivity, Max-Planck-Institute for Human Cognitive \\ and Brain Sciences, Leipzig, Germany \\ ${ }^{3}$ Institute of Philosophy, Free University Berlin, Berlin, Germany
}

\begin{abstract}
The term "connectome" is commonly taken to describe a complete map of neural connections in a nervous system of a given species. This chapter provides a critical perspective on the role of connectomes in neuroscientific practice and asks how the connectomic approach fits into a larger context in which network thinking permeates technology, infrastructure, social life and the economy. In the first part of this chapter, we argue that, seen from the perspective of ongoing research, the notion of connectomes as "complete descriptions" is misguided. Our argument combines Rachel Ankeny's analysis of neuroanatomical wiring diagrams as "descriptive models" with Hans-Jörg Rheinberger's notion of "epistemic objects", i.e. targets of research that are still partially unknown. Combining these aspects we conclude that connectomes are constitutively epistemic objects: there just is no way to turn them into permanent and complete technical standards because the possibilities to map connection properties under different modeling assumptions are potentially inexhaustible. In the second part of the chapter, we use this understanding of connectomes as constitutively epistemic objects in order to critically assess the historical and political dimensions of current neuroscientific research. We argue that connectomics shows how the notion of the "brain as a network" has become the dominant metaphor of contemporary brain research. We further point out that this metaphor shares (potentially problematic) affinities to the form of contemporary "network societies". We close by pointing out how the relation between connectomes and networks in society could be used in a more fruitful manner.
\end{abstract}

KEYWORDS: Connectome, philosophy of neuroscience, epistemic object, network society, critical neuroscience 
Penultimate draft to appear in: N. Rose, T. Mahfoud, \& S. McLean (eds.). Modeling Brains: The Making and Use of Animal Models in Neuroscience and Psychiatry. Progress in Brain Research 233, Elsevier.

\section{Introduction}

About a decade ago, Sporns et al. (2005) introduced the term "connectome" to describe the effort of assembling a "comprehensive structural description of the network of elements and connections forming the human brain" (ibid., 0245, see Sporns 2013 and Catani et al. 2013 for historical overviews). The suffix "ome" was inspired by earlier projects that mapped the complete structure of entities in a particular biological domain-most notably the Human Genome Project. In subsequent years, several small- and large-scale projects that map connectomes in humans and model organisms have been launched (e.g., Lu et al. 2009, Van Essen et al. 2013a, Oh et al. 2014, Kasthuri et al. 2015). Despite notable advancements, it remains an open question whether connectomics can provide complete descriptions or comprehensive maps of neural connections of complex nervous systems. Yet, connectomics has already provided researchers with new conceptual and methodological tools. In particular, computational neuroanatomist Olaf Sporns $(2011,2012)$ popularized the idea that the brain is a complex network that can be studied with the tools of mathematical graph theory - a trend that also reflects the increasing prominence of networks in science and society more generally.

In this chapter, we pose two questions about the connectomic approach: What role do connectomes play in neuroscientific practice? And given this role, how does the connectomic idea of the "brain as a network" fit into a larger historical and social context? Although these questions may at first seem independent from each other, we think they belong together because the exchange with social and cultural ideas is a vital part of the dynamics of scientific research. Our answers to these questions are intended to provide a critical perspective on connectomics in the dual sense of "critique". In the Kantian sense of critique, we analyze and distinguish the prospects and limits of contemporary connectomics research. In the sociopolitical sense of "critique", we interrogate the emergence of connectomes at a time where networks are suddenly "everywhere": in nature, technologies, infrastructures, social life and the economy. By combining these approaches, we hope to provide neuroscience practitioners with analytical tools to reflect on their own research, and to motivate science and society scholars to investigate one of the most rapidly progressing branches of brain research, whose wider ramifications we can only sketch in outline.

In the first part of the chapter we argue that the notion of connectomes as "complete descriptions" or "parts lists" seriously misconstrues the role that connectomes play in 
Penultimate draft to appear in: N. Rose, T. Mahfoud, \& S. McLean (eds.). Modeling Brains: The Making and Use of Animal Models in Neuroscience and Psychiatry. Progress in Brain Research 233, Elsevier.

neuroscientific research. Methodologically, our thesis relies on the approach of philosophy of science in practice, which focuses on the prospective role of evidence, methods and models in ongoing research (Ankeny et al. 2011, Rouse 2015), rather than the retrospective interpretation of established scientific knowledge. ${ }^{1}$ Our main message is that from the perspective of ongoing research, connectomes are not complete descriptions but revisable normative standards for further experimentation and modeling. We claim that this is even the case for the wiring diagram of the nematode worm C. elegans, which is widely regarded as being completed by White et al. (1986). Using Rachel Ankeny's notion of a "descriptive model", we show that the 1986 wiring diagram presents only one stage in an ongoing process of creating and revising the standards for further experimentation and modeling (section 1.1). We then apply this insight to current connectomics approaches at the macro-, meso- and microscale (section 1.2). While Ankeny's notion of "descriptive model" is helpful to understand the role of idealization and modelling assumptions in these approaches, it does not characterize how researchers use different measurement signals to trace various connection properties. We claim that this signal-property relation qualifies connectomes as what Hans-Jörg Rheinberger has called "epistemic objects", i.e. targets of ongoing research whose characteristics are not yet fully known (section 1.3). Combining Ankeny's and Rheinberger's analyses, we conclude that connectomes are constitutively epistemic objects: they can never be turned into permanent and complete technical standards because the possibilities to map connection properties under different modeling assumptions are potentially inexhaustible.

In the second part of the chapter, we use this notion of connectomes as constitutively epistemic objects to critically assess the historical and political dimensions of current neuroscientific research. Contrary to historian of neuroscience Cornelius Borck who claims that the Internet is no central metaphor to understand the brain, we argue that connectomics shows how the Internet - and the concept "network" more generally — has indeed become a central and fruitful metaphor in contemporary brain research (section 2.1). Drawing on Borck's own analyses we show how the "brain as network" metaphor shares affinities to the form of contemporary "network societies" which emerged in post-Fordist economies. We highlight how this affinity can become problematic when it is used to naturalize potentially harmful forms of social existence (section 2.2). We close by sketching "model domain realism" as a way to link connectomes and network societies in a less problematic and more fruitful manner (section 2.3). 


\section{Connectomes in neuroscientific practice}

Connectomes are often defined as "inventories" or "parts lists" that completely describe the entities in the domain of neural connectivity. According to Sporns (2013), this definition derives from the features that connectomes share with other "omes": "universality (they apply to a broad range of systems and species), totality (they comprise a complete and finite set of data), and permanence (they remain valid as knowledge continues to grow)" (ibid., 55, see also Sporns 2012, 8). Sporns' definition suggests that connectomes are constructed by recording a complete dataset of neural connections which in turn reveals species-invariant connectivity principles, thus rendering connectomes capable to serve as permanent standards for future research. In this part of our chapter we argue that this view does not adequately characterize how connectomes are in fact generated and used in neuroscientific practice.

\subsection{The C. elegans wiring diagram as a descriptive model}

We begin our analysis with the connectome of the nematode worm C. elegans. It is common parlance in neuroscience circles that the nervous system of $C$. elegans has been completely mapped three decades ago (e.g., Lichtman and Sanes 2008, 347, Sporns 2012, 27, Catani et al. 2013, 11, Oh et al. 2014, 207). In a book-length study known as “The Mind of a Worm”, White et al. (1986) used electron microscopy (EM) to map the worm's 302 neurons and their synaptic connections. We will show that the construction, status and role of the 1986 wiring diagram are hard to reconcile with Sporns' definition of connectomes as total, universal, and permanent. We argue instead that mapping the $C$. elegans connectome is better understood as an ongoing process to create revisable normative standards for further experimentation and modeling.

The construction of the $C$. elegans wiring diagram first shows that connectomes are not simply finite data sets — as specified in the totality dimension of Sporns' definition — but represent a special kind of model. Ankeny (2000) argues that White et al. (1986) used idealization and abstraction as modeling steps to move from the recorded electron micrographs to the general wiring diagram. Their final dataset consisted of overlapping sections from four different worms which provided clearest visualizations of connectivity. The use of data from several individuals was justified by two idealizing assumptions. First, since the researchers aimed to uncover the canonical 
nervous system of the nematode worm, invariance in connectivity was highlighted and variance ignored. Second, because canonical connections were assumed to occur between neuronal types, the final rendering of the diagram was further simplified by sorting the 302 nerves cells into 118 classes. Researchers could now neglect differences between single cells within a class, because they represented tokens of the same type (cf. ibid., S264). Ankeny concludes that this idealization process resulted in a wiring diagram that is in fact abstracted from the natural entity C. elegans. She therefore calls this wiring diagram a descriptive model: Its purpose is not to test a particular theory or hypothesis that would explain the functioning of nematode nervous systems. Rather, its purpose is to disclose the canonical structure of such nervous systems against which further experimental data or theoretical hypotheses can be compared. Emphasizing the descriptive modelling steps illustrates why the $C$. elegans connectome is not identical to the finite dataset that White and colleagues recorded.

The construction of the c.elegans wiring diagram secondly shows that the presumed "completeness" of a connectomic dataset is a function of technological possibility. Because White et al. (1986) reconstructed long axons by interpolating between several micrographs continuous connectivity data was missing for the mid-body of the worm. Going back to the laboratory notebooks of White and colleagues, Varshney et al. (2011) also discovered that no high-power electron micrographs existed for a large, dorsally located region. To alleviate these issues, Varshney and colleagues updated or added of over 3000 synaptic contacts between chemical and electrical synapses as well as neuromuscular junctions. Yet, even this updated connectome is considered to be only $90 \%$ complete, with electrical synapses posing a particularly difficult case. Electrical synapses are difficult to identify with serial EM, because their 3D-structure is obscured when tissues are cut into thin sections. Only recently has it become technically possible to identify such synapses more directly with improved preparation protocols and super-resolution array tomography (Markert et al. 2016). This example illustrates that what counted as a breakthrough to completion in the past appears as highly incomplete from the technological viewpoint of today.

Although the 1986 wiring diagram is idealized and technically limited, Ankeny (2000) argues that this descriptive model had a canonical status: certain features of the diagram could apply to a broader range of species and systems (this is the 'universality' feature in Sporns' definition). The discussion above suggests that a complete data set is not required to achieve this 
Penultimate draft to appear in: N. Rose, T. Mahfoud, \& S. McLean (eds.). Modeling Brains: The Making and Use of Animal Models in Neuroscience and Psychiatry. Progress in Brain Research 233, Elsevier.

status. Rather, the C. elegans connectome from 1986 became canonical because its construction was based on what we call the fundamental presupposition of connectomics:

The functional properties of a nervous system are largely determined by the characteristics of the component neurons and the pattern of synaptic connections between them (White et al. 1986, 2).

The fundamental presupposition is the main reason why today's researchers see themselves as continuing the work of the early C. elegans anatomists (Sporns et al. 2005, Lichtman and Sanes 2008, Varshney et al. 2011, Oh et al. 2014). The significance of this unifying role may well outrun the extent of completeness of a given connectome. The 1986 wiring diagram played exactly this unifying role because it represented both a "consensus state of the worm in terms of its neural pattern and connections [and a] prototype not only of the nervous system of C. elegans, but more generally of a simple metazoan nervous system" (Ankeny 2000, S262). In virtue of the fundamental presupposition that structure determines function, the utility of the descriptive model is not primarily evaluated according to its completeness with regard to C. elegans, but according to its prototypical status with regard to the domain of neural connectivity.

For the wiring diagram to play a role as prototype in scientific practice, its use needs to be independent from the initial circumstances under which it was constructed. Ankeny (2000) therefore argues that the wiring diagram is a "phenomenon isolated from a larger dataset" (ibid., S261). ${ }^{2}$ The observed data are the micrographs recorded by electron microscopes, whereas the phenomenon is the canonical nervous system presented in the form of the wiring diagram:

[I]nherent in the notion of a descriptive model is the recognition of a pattern or set of patterns which correspond to fundamental processes. These patterns or phenomena can then serve as the basis for further development of explanations and ultimately theories (ibid., S269).

Because the wiring diagram displays a pattern or phenomenon, it mediates between anatomical experiments that generate the descriptive content as data and the explanations and theories which use the data as evidence (on models as mediators, see Morgan and Morrison 1999). Descriptive models play such a mediating role because they specify how researchers should reason about neural connectivity. We therefore argue that the primary role of descriptive models is to serve as a normative standard for inferential reasoning about different aspects of neural connectivity, such as comparing different connectivity patterns and making inferences from structure to neural function or organismal behavior. The role of $C$. elegans wiring diagram as a normative standard is thus twofold. In virtue of its prototypical status, it can be used to determine whether further 
Penultimate draft to appear in: N. Rose, T. Mahfoud, \& S. McLean (eds.). Modeling Brains: The Making and Use of Animal Models in Neuroscience and Psychiatry. Progress in Brain Research 233, Elsevier.

anatomical reconstructions of individual worms are correct or incorrect, and in virtue of its canonical status and the fundamental presupposition, it guides researchers to understand how structure relates to function in the domain of neural connectivity more generally. ${ }^{3}$

The role of descriptive models as normative standards also alters the extent to which connectomes can be permanent standards for future research. Ankeny (2000) points out that the reliance on the fundamental presupposition makes such standards empirically revisable:

[Descriptive models] will need to be altered as new experimental approaches reveal [their] limitations; at the extreme, alterations in the descriptive model will result in changes in the idealized presuppositions behind the description (in this case that the functional properties of a nervous system are determined directly by its structure) (ibid., S266, emphasis in original).

In order to illustrate the revisability of descriptive models, consider how the 1986 wiring diagram has been used to draw inferences from connectivity structure to the neural functions of C. elegans. While the 1986 wiring diagram helped researchers to relate specific neuron types to worm behavior (e.g., touch-avoidance response, egg-laying), it did not allow them to infer which synaptic connections were inhibitory or excitatory (cf. Bargmann and Marder 2013, 485). Consequently, later connectomics researchers concluded that structural connectivity is necessary but by itself insufficient to determine neural functions (cf. Varshney et al. 2011, 1). This example illustrates how further experiments made possible by the wiring diagram can alter the fundamental presupposition behind its construction.

In sum, even in the case of $C$. elegans, the notion of a connectome as a complete map of neural connections is at odds with the way wiring diagrams are constructed by idealizing individual nervous systems to arrive at abstract descriptive models. Such diagrams can only play the role of a normative standard because they embody assumptions about $C$. elegans as a prototype for metazoan neural connectivity and because they are of (limited) use for inferring functions from structure. As a normative standard, the $C$. elegans connectome is revisable in light of the experimental results it enables and incomplete with respect to technological innovations that supersede the methodologies employed in its construction. In the next section, we show that these features of descriptive modeling also apply to contemporary connectomics approaches. 
Penultimate draft to appear in: N. Rose, T. Mahfoud, \& S. McLean (eds.). Modeling Brains: The Making and Use of Animal Models in Neuroscience and Psychiatry. Progress in Brain Research 233, Elsevier.

\subsection{Descriptive modelling in contemporary connectomics}

Since the publication of Ankeny's analysis, there has been an explosion of connectomic research using different modeling approaches to describe neural connectivity. In order to demonstrate that the philosophical analysis developed so far can be extended to these approaches, consider the following take on the completeness of connectomes:

In my standard talks I say that the connectome is meant to be "comprehensive"-in quotation marksbecause there is a macro-connectome, there is a meso-connectome and there is a micro-connectome, and none of them being comprehensive (David van Essen, June 16 2015, Meeting of the Organization for Human Brain Mapping, Honolulu, HI, USA). ${ }^{4}$

Van Essen, one of the principal investigators in the Human Connectome Project (HCP), points out here that connectomes today are studied at different scales of inquiry. The scale of a connectome depends both on the precision of instruments for data acquisition and on the size of neural entities (areas, circuits, neurons) whose interconnections are to be mapped. Thus, from the practitioner's perspective contemporary connectomics consists of a plethora of species-specific, scale-dependent and partial connectomes. In the following, we will use the distinction into macro-, meso- and microscopic scales to analyse descriptive modelling in contemporary connectomics.

Consider first the role of idealizing assumptions in different connectomic approaches. In contrast to the idealizing assumption in the C. elegans connectome, the HCP does not eliminate, but in fact aims to investigate the variability of human brain connectivity with diffusion MRI (dMRI). We will discuss this method to measure connectivity in detail in the next section. What is important for now is that the HCP spent about half of its funding period to optimise its dMRI acquisition, pre-processing and tractography protocols for a customized MR scanner (Van Essen et al. 2013a). This optimization is deemed necessary to detect subtle variations in connectivity between individuals. To ensure sufficient variation in their dataset, the HCP also aims to recruit 1200 healthy adult twins and their non-twin siblings that represent the ethnographic distribution of the US population according to the 2000 census. This participant group obviously does not capture the full extent of human brain variability. The focus on this group represents an idealizing assumption in macroconnectomic modeling. The assumption is justified both by economic constraints and the HCP's interest in genetic and environmental influences on variance in brain connectivity. In contrast to the C. elegans projects discussed above, however, the HCP researchers 
Penultimate draft to appear in: N. Rose, T. Mahfoud, \& S. McLean (eds.). Modeling Brains: The Making and Use of Animal Models in Neuroscience and Psychiatry. Progress in Brain Research 233, Elsevier.

do not use their idealizing assumption to move from connectivity data to a species-general wiring diagram. ${ }^{5}$ Ankeny argues that the $C$. elegans wiring diagram was necessary to make the information contained in the EM slices portable between laboratories working on nematode nervous systems. This is not required for the HCP MRI data which are freely available online in raw and preprocessed form. Because their data are already portable, the HCP could record a connectomic dataset of idealized variability independently from constructing a wiring diagram of macroscale human brain connectivity.

Portability is also a main feature of the Allen Mouse Brain Connectivity Atlas (Oh et al. 2014). The data of this mesoscale connectome are made available via the neuroinformatic infrastructure of the Allen Brain Institute (ABI), and structures within the dataset are annotated according project's ontology of the mouse brain. Like the HCP, the ABI also emphasizes standardized, large-scale acquisition of high-quality and openly accessible data, but their mesoscale connectome does not stress variability. Instead, Oh et al. (2014) aimed to create a template brain that does not contain inhomogeneities within a region or variability of inter-regional connectivity between individuals. As we will detail below, these researchers used viral tracers instead of EM to map mesoscopic instead of microscopic connectivity, but their approach otherwise closely resembles the C. elegans research discussed above. Oh et al. (2014) also provide a data set, a classification of the connection data according to injection and termination site (connectivity matrix), and a wiring diagram between cortical and thalamic regions that accords to this classification.

In contrast to the $C$. elegans and the Allen mouse connectome, some current microconnectomic approaches do not eliminate or even idealize variability. For instance, Lu et al. (2009) used confocal laser scanning microscopes and fluorescent staining to image every individual axonal branching pattern of the interscutularis muscle in mice. The small number of axons allowed the researchers to investigate the "absolute" variability of neuronal connections in this structure. No attempt was made to generate a canonical wiring diagram of the muscle, because each axonal branching pattern possessed a distinct topology, both within and between animals. Although idealization may play a somewhat lesser role microconnectomic descriptive modeling, we do not think that such studies are completely unbiased or purely observational. In fact, a rather strong idealizing assumption implicit in many microconnectomic projects so far is that connections formed by glia cells — either with other glia cells or with neurons — can be excluded from microscale 
Penultimate draft to appear in: N. Rose, T. Mahfoud, \& S. McLean (eds.). Modeling Brains: The Making and Use of Animal Models in Neuroscience and Psychiatry. Progress in Brain Research 233, Elsevier.

connectomes. While this assumption greatly simplifies the already daunting task to map connectivity at the resolution of nanometers, it obscures the fact that glia cells shape neuronal connectivity both structurally and functionally (Fields et al. 2015).

The analysis of contemporary connectomics reveals that the type of idealization used in descriptive modeling depends on the target of connectomic mapping. By "target" we mean a feature of brain connectivity that can be highlighted or supressed by the idealization strategy used in a particular connectomic mapping approach. ${ }^{6}$ Consider, for instance, the feature that connectivity varies between individual brains of the same species. With regard to variability, idealization can range from eliminating variance (Allen meso-connectome), over investigating a selected range of variability (HCP macro-connectome) to displaying "absolute" variability (interscutularis muscle connectome). Each idealization strategy displays distinct advantages and drawbacks that depend both on the target and the research interests of connectomic researchers (e.g., providing a dataset that captures variability, creating a canonical wiring diagram, or reconstructing individual axonal branches). The completeness of the resulting connectomes needs to be evaluated relative to these research interests and the idealizing assumptions used to pursue them.

This plurality of descriptive modeling is further amplified by the scale-dependent modeling assumptions that supplement the fundamental presupposition of connectomics. Researchers need such additional assumptions to reveal the structural connection properties which (in part) determine neural function. In macroconnectomics, dMRI data first need to be transformed into a connection matrix that parcellates the human brain into distinct regions and assigns connection probabilities between each of these regions. Researchers then interpret the connection matrix as composed of nodes and edges to calculate abstract network properties with tools from mathematical graph theory. Such network properties can range from the number of connections of a single node (degree), over the existence of densely intra- and sparsely interconnected subgraphs (modules) to the estimation of complex topologies of the entire network (Sporns 2011, 2012). To infer constraints about human brain function from their graph theoretical calculations, researchers usually assume that mathematical topologies (i) reflect the spatial organization of the human brain, (ii) illuminate the flow of information in the anatomical network, and (iii) can be interpreted in terms of wiring costs of the underlying structure. Without these assumptions, macroscale connectivity data do not reveal the network structure of the human connectome. 
Penultimate draft to appear in: N. Rose, T. Mahfoud, \& S. McLean (eds.). Modeling Brains: The Making and Use of Animal Models in Neuroscience and Psychiatry. Progress in Brain Research 233, Elsevier.

Although Oh et al. (2014) also calculated graph theoretical measures of the mesoscale mouse connectome (e.g., small-world index), these mathematical operations depend on two scale-specific assumptions about inter-regional connectivity. The researchers first assumed regional homogeneity to justify the use of partial tracer injections as a stand-in for the overall pattern of connectivity within a particular brain region. They secondly assumed projection additivity such that the projection densities observed in the staining data could be linearly extrapolated to unstained regions and unobserved connection patterns. These assumptions are specific to the staining protocol that regulates data acquisition at the mesoscale of inter-regional connectivity. Consequently, the connectivity matrix in Oh et al. (2014) is constructed from these assumptions, which is why they also make the calculation of topological network properties possible. So despite similar mathematical appearance, graph-theoretical measures in macro- and mesoconnectomic approaches can rely on different descriptive modeling assumptions.

Microconnectomics researchers sometimes use specific modeling assumptions to simplify the task of describing complex synaptic connection patterns. Lu et al.'s (2009) approach of displaying every axonal branching topology is unfeasible for virtually every connectome that exceeds the few connections of the interscutularis muscle. Microconnectomics researchers therefore often search for motifs that are overrepresented in the connectivity data when a given connectome is compared to a randomly connected graph. For instance, Varshney et al. $(2011,9)$ found that fully connected triplets as well as "fan" and "diamond" motifs are statistically overrepresented in the C. elegans network of chemical synapses. Although the concept of network motifs itself is not specific to microconnectomics (cf. Sporns 2011, 12, 101), Varshney et al.'s analysis is scale-dependent because it operates over the synaptic connectivity pattern of individual neurons. Their structural approach does not allow for the omission of connections outside the motif, a practice that is common when researchers draw functional circuit diagrams which specify the contribution of interconnected neurons to behaviorally relevant information processing (cf. Varshney et al. 2011, 9).

We have shown in this section that the application of Ankeny's analysis to contemporary connectomics reveals two additional aspects of descriptive modeling in neuroanatomy. First, we have pointed out that the types of idealization that researchers use depend on the target of connectomic mapping. The completeness of the resulting connectomic maps has to be evaluated with regard to the feature of connectivity these researchers are interested in. We secondly argued 
Penultimate draft to appear in: N. Rose, T. Mahfoud, \& S. McLean (eds.). Modeling Brains: The Making and Use of Animal Models in Neuroscience and Psychiatry. Progress in Brain Research 233, Elsevier.

that without scale-dependent modeling assumptions, connectomics researchers are unable to reveal network properties from the requisite connectivity data. These network properties in turn allow them to draw inferences about the functions of the interconnected neural entities. Ankeny has pointed out that the $C$. elegans wiring diagram is revisable with regard to the fundamental presupposition that structure directly determines function. Our analysis in this section implies that connectomes that are constructed today are revisable along several dimensions that depend on research interest and idealization strategy. In the next section, we will elaborate on this multidimensionality by adding another factor that is missing from Ankeny's analysis.

\subsection{Connectomes as constitutively epistemic objects}

We have seen that connectomes are currently mapped at different scales using different measurement technologies such as dMRI, viral tracers or EM. Yet, Ankeny's notion of a descriptive model does not provide the analytical tools to understand how the signals of these measurement devices relate to the neural properties that connectomic researchers want to investigate. In this section, we argue that this signal-property relation qualifies connectomes as “epistemic objects” (Rheinberger 1997). In order to do so, we begin by highlighting how different measurement signals used in connectomics focus on different aspects of connectivity.

As mentioned above, macroconnectomics relies on dMRI, whose signal traces water diffusion, i.e. how water molecules move through three-dimensional cubes of brain tissue (voxels). Because fiber bundles hinder water to diffuse in all directions, they will bias the dMRI signal in a voxel. From this bias, researchers can estimate the location of the bundles from the main direction of diffusion across voxels (e.g., by calculating the fiber orientation density function, cf. Craddock et al. 2013, 528). As a result, researchers can reconstruct white matter pathways throughout the brain. The reconstructed pathways do not represent individual axons but rather approximate the main direction of major fibre bundles. Because the reconstruction usually operates over white matter voxels only, dMRI focuses on long-range connections between cortical areas, at the expense of ignoring short connections between grey matter voxels. Even within white matter voxels, however, the dMRI signal can result in ambiguities. Fibers that bend, disperse, cross or "kiss" each other within a voxel can easily be missed or inaccurately modeled (cf. ibid., 529). Compiling a complete and accurate matrix of distant but connected cortical areas is also complicated by the 
Penultimate draft to appear in: N. Rose, T. Mahfoud, \& S. McLean (eds.). Modeling Brains: The Making and Use of Animal Models in Neuroscience and Psychiatry. Progress in Brain Research 233, Elsevier.

"gyral bias" of dMRI (Van Essen et al. 2013b). Since the endpoints of reconstructed pathways predominantly lie in the crowns of the folded cortical surface (gyri), dMRI is currently unsuited to accurately map fiber bundles that terminate in the deep furrows (sulci) of the cortex.

In contrast to noninvasive dMRI, mesoconnectomics often uses viral vectors to invasively trace inter-regional connections in animal models. For instance, Oh et al. (2014) combined the adeno-associated virus (AAV) with gene promoter synapsin I. When injected into mouse brains, this viral vector causes the neurons to glow bright green, because they start to produce enhanced green fluorescent protein. This method allows for high-throughput anterograde tracing, i.e. the simultaneous mapping of many neural connections from the cell body to the synapse. Yet, it also comes with drawbacks in resolving certain connection properties. First, Oh et al. (2014) used relatively large injection sites which reveal connections that cross boundaries between cortical or subcortical areas. Second, although the AAV tracer labels synaptic terminals, these can only be manually distinguished from axonal fibers that pass through an area without connecting to other neurons (cf. ibid., 213). The resulting connectivity matrix inherits this indistinguishability and also contains false positives from segmentation artefacts. Thirdly and finally, the AAV tracer could contain biases and therefore not label all neuronal cell types equally. Nathanson et al. (2009) reported that $\mathrm{AAV}$ combined with a $\mathrm{CAG}$ promoter preferentially labels inhibitory neurons, although the human synapsin I promoter seems to eliminate this bias when AAV is injected at very high concentrations. Since Oh et al.'s gene construct contains both promoters, it is difficult to assess whether their viral tracer is biased towards inhibitory neurons or not. What should become clear, however, is that the choice of a particular viral gene construct for mesoscopic mapping represents a trade-off between acquisition speed, coverage and accuracy of the interregional connections that are to be traced.

Measurements at the microconnectomic scale are also characterized by a trade-off between speed, coverage and accuracy when tracing the synaptic connections of individual neurons. EM provides the resolution to image synaptic gaps directly, but the reconstruction of entire neurons and their synaptic contacts is time-consuming and difficult to automate. Microconnectomic EM approaches therefore remain limited to small volumes of cerebral tissue, making it unlikely to map entire microconnectomes in the near future (Kashturi et al. 2015). In contrast, experiments that use light microscopy (LM) can reconstruct dense neuronal populations much faster. For instance, the 
Brainbow technique uses LM to image individually colored neurons and connections. ${ }^{7}$ Although Brainbow allows a quicker reconstruction of dense connectivity structures, the actual synaptic contacts have to be inferred because they are smaller than what conventional LM can resolve (Yook et al. 2013, 4751). This technical limitation of has recently been tackled by the development of super-resolution LM. ${ }^{8}$ Yet, even such improved LM methods occasionally record false positives, which makes the estimation of absolute synapse numbers difficult (cf. ibid.). In light of this complex landscape of measurement methods, it becomes clear that each signal traces synaptic connections by measuring different properties. None of these methods is by itself sufficient to map microconnectomes in complex organisms so far, although their differential combination could bring the field closer to this objective in the future.

We have detailed the different connectomic measurement methods here to highlight that each signal indexically traces another connection property. An index is a sign that can stand in a material relationship to the object or property it signifies, e.g. smoke can be an index of fire. Similarly, signals measured in connectomics can be an index for connection properties. Water diffusion measured by dMRI can be an index for long-range fiber bundles crossing the cortex. Green fluorescent protein expressed by the AAV tracer can be an index of inter-regional axonal connections. Vesicle-like structures in an EM image can be an index for a synaptic terminal, and differently colored lines in a section from a transgenic Brainbow mouse brain can be an index for axons from distinct neuronal cell bodies. We put this indexical relationship between signal and property in conditional form because every index is only an indirect sign of the connection property it signifies. The success conditions of the indexical relationship in these cases include the researchers' ability to rule out artefacts such as labeling biases, image distortions or insufficient resolution that were outlined above. Because indexical and artefactual relations usually co-exist in experimental practice it is often difficult to tell whether an unexpected result simply represents a measurement error or a previously unknown connection property. Consider again the "gyral bias" in dMRI measurements discussed above. Sorting indexical from artefactual relations in this surprising deviation from classical tracer results depends on whether axon fibers enter grey matter at the right angle or gradually "peel off" at oblique angles until a few reach the gyral crown (cf. Van Essen et al. 2013b, 348). Similar questions arise between the connections that microconnectomic signals reveal and the statistical model assumptions that guide connectivity reconstruction. Is stochastic "randomness" a property of synaptic connections or does it reflect our 
Penultimate draft to appear in: N. Rose, T. Mahfoud, \& S. McLean (eds.). Modeling Brains: The Making and Use of Animal Models in Neuroscience and Psychiatry. Progress in Brain Research 233, Elsevier.

current inability to map connectivity at the cellular and subcellular scale (cf. Sporns 2011, 71, Kashturi et al. 2015, 658)? Answering questions such as these requires further experimentation in which researchers eliminate artifacts and try to validate connection properties that so far remain partially unknown.

The historian of science and molecular biologist Hans-Jörg Rheinberger has called these composites of partially unknown properties and indexical traces from measurement signals epistemic objects (Rheinberger 1997). Rheinberger introduced this concept in his historical case study of protein synthesis research between 1947 and 1961. His micro-analyses of experimental practice (especially that of Paul Zamecnik's group at MGH and Harvard) showed how the mechanism of protein synthesis emerged as a research focus in cancer biology, before becoming the explicit target of biochemical in vivo and in vitro experiments, which were gradually supplemented by molecular biological ideas about the genetic code and information transfer. In this multi-disciplinary research context, new entities were often unexpectedly discovered under experimental circumstances that were initially geared towards a different aspect of the protein synthesis mechanism. Because such emerging entities "embody what one does not yet know" (ibid., 28), Rheinberger calls them "epistemic objects". An example of an epistemic object from his case study is what is now called "transfer-RNA". This entity first appeared as an ineliminable contamination in the residual fraction of centrifuged rat liver cells. Around 1957, Zamecnik was searching for an activity that would synthesize RNA in this rat liver system. Instead, he found a small, already synthesized RNA molecule, which he defined operationally as "soluble RNA" and functionally as an intermediate product of protein synthesis (cf. ibid., 154f.). Three years after this discovery, Richard Schweet proposed the term "transfer-RNA", which suggested that it played a role in carrying amino acids and transferring genetic information (cf. ibid., 189). This episode illustrates a typical - though not the only possible - trajectory of an epistemic object: from noise in the experimental system to an operationally and later functionally defined entity. Like in the connectomic examples above, protein synthesis researchers had to determine which experimental signals were artefactual and which were indexically related to the target of research. In this process, "the scientific object is cast into a traceable configuration" (ibid., 110). In other words, epistemic objects are composed of measurement signals that can indexically trace (partially) unknown properties within an experimental system. 
Penultimate draft to appear in: N. Rose, T. Mahfoud, \& S. McLean (eds.). Modeling Brains: The Making and Use of Animal Models in Neuroscience and Psychiatry. Progress in Brain Research 233, Elsevier.

With the index-property relation in connectomics and Rheinberger's analysis in place, we can now see why connectomes qualify as epistemic objects. Contemporary connectomics resembles the protein synthesis research of Rheinberger's case study since it brings multiple (neuro)scientific disciplines together. Depending on the target of research, the successful investigation of connectomes requires experts in neuroanatomy, MR-physics, graph theory, viral genetics, neuroinformatics or developmental biology, just to name the more obvious ones. Like in protein synthesis research, each scientific group investigates their target of connectomic mapping with a particular experimental system. Microconnectomic researchers, for instance, speak of the "Brainbow system" when investigating a partially unknown connection property, such as whether multiple retinal terminals that connect to one geniculate synapse descend from the same or different cells in the retina (Hammer et al. 2015). Within such a system, the target of research is "epistemic" because the connection property that is being traced is partially unknown. Yet this target is also object-like because the signal can stand in a material relationship to the connection property. The investigation of such epistemic objects is made possible by various technical objects such as gene constructs, sections from transgenic mice and light microscopes in the case of Brainbow, or MR scanners as well as reconstruction algorithms and fiber orientation density functions in the case of macroconnectomic tractography.

We are now in a position to articulate a different picture of contemporary connectomics. Our addition of the indexical signal-property relation grounds Ankeny's analysis of connectomes as descriptive models more firmly in experimental practice. The descriptive modeling steps of idealization, abstraction, and the use of (scale-specific) modeling assumptions to create connectivity matrices and wiring diagrams are all based on the signal-property relationship that is embodied in a specific connectomic experimental system. We therefore think that it is appropriate to characterize connectomes as epistemic objects, without thereby denying the importance of modeling steps outlined in the previous sections. In fact, combining Ankeny's and Rheinberger's analyses provides an alternative definition of connectomics. According to this definition, connectomics is the practice of indexically tracing partially unknown connection properties under model assumptions to create idealized and revisable diagrams of brain connectivity. This definition stands in strong contrast to Sporns' definition that we quoted in the beginning of this part of the chapter. Sporns defined connectomics as the recording of complete and finite datasets of structural connectivity that reveal universal and permanent principles of brain connectivity. Drawing on 
Penultimate draft to appear in: N. Rose, T. Mahfoud, \& S. McLean (eds.). Modeling Brains: The Making and Use of Animal Models in Neuroscience and Psychiatry. Progress in Brain Research 233, Elsevier.

Rheinberger we can now see that this definition characterizes connectomes as technical objects. Sporns' view suggests that connectomes are permanent standards which enable experiments on other epistemic objects such as unknown neural functions that are partially determined by the connectivity structure of a nervous system. This view is not entirely mistaken from the perspective advocated here. The distinction between epistemic and technical objects is a function of how a particular dataset, method or model is used in an experiment. On Rheinberger's account, a scientific field develops by transforming its epistemic objects into technical objects that can then be used instrumentally in subsequent experiments (cf. Rheinberger 1997, 21, 28f., 80, 110, 141). In fact, the connectomes that we reviewed here have been and are used as technical objects in the sense that researchers take the datasets or wiring diagrams to investigate neural functions or principles of wiring (Marder and Bergmann 2013, Shine et al. 2016, Goulas et al. 2016). Yet, we will now draw on the insights from the previous sections to claim that connectomes are constitutively epistemic objects that for principled reasons cannot be turned into complete and permanent technical standards.

The first step in our argument is based on the premise that the term "connectome" simultaneously refers both to a structure of the brain - the totality of elements and their connections - and the model that describes this structure. This simultaneous reference implies that connectomics researchers must adjudicate between what we will call object factors and research factors when investigating neural connectivity. One object factor is that connectomes are objects that are in a sense "defined" by their variability, both over time and between individuals. This variability leads to the research factor of having to choose an idealizing assumption with regard to the target of connectomic mapping (section 1.2). Another object factor is the existence of multiple scales at which the connectome in a particular species can be investigated. By choosing a scale, researchers have to determine two research factors, namely choosing scale-dependent modeling assumptions and choosing a measurement signal that indexically traces the connection property upon which the connectome is going to be constructed.

The second step in our argument is that object factors and research factors always stand in a tension that can be differently resolved depending on which method of modeling and measurement is chosen. In the previous sections, we only surveyed a small sample of possible solutions to map variability, infer function from structure or create wiring diagrams. We suspect that the space of possible solutions is potentially inexhaustible, and that there exists no optimal set of research 
Penultimate draft to appear in: N. Rose, T. Mahfoud, \& S. McLean (eds.). Modeling Brains: The Making and Use of Animal Models in Neuroscience and Psychiatry. Progress in Brain Research 233, Elsevier.

factors that meets all the object factors equally while still being useful for further experimentation. It is because of the myriad ways in which object and research factors can be combined that we think that connectomes cannot be completed. Because they are constitutively epistemic objects, it is inadequate to think of connectomes as "parts lists" like the ones a car mechanic uses to find the broken part in a malfunctioning motor. This analogy to "parts lists" or "inventories" is often made on the assumption that the structure of a nervous system consists of a finite set of elements and their connections (cf. Sporns 2012, 31). We think that the analogy mistakenly assumes that there exists only one fixed referential relationship between the connectome as an object and the connectome as a descriptive model. If the reference were fixed, then there would exist a finite set of datapoints that, if recorded, would correspond exactly to the structure. We pointed out earlier, however, that connectomics researchers use multiple signals that indexically trace different connection properties. Each of these indices possesses a different referential relationship to a structural aspect of connectomes. And given that the technologies upon which these indices are based will further develop in the future, these referential relationships will further change rather than stay fixed. Therefore, the possibilities of creating usable, highly valid connectomic datasets and models are as infinite as the combination of object and research factors is inexhaustible. The notion of a finite connectomic dataset with a fixed reference rests on a static picture of neuroscientific research. Our notion of connectomes as constitutively epistemic objects can fully account for the dynamics by which brain research progresses, whereas the notion of connectomes as complete descriptions cannot.

The third and final step in our argument is that even if researchers regard a particular descriptive model as "complete", the concept of the connectome itself possesses an open texture. The concept "connectome" is open because although it demands connectivity descriptions to be "complete", the degree of completeness of a particular descriptive model and its significance for understanding function always depends on the specific indexical traces and scale-dependent modeling assumptions used to construct that model. Moreover, what it means to give a "complete" connectomic description will be re-evaluated once researchers apply a particular descriptive model in new experiments that investigate the relationship between neural structure and function. An instructive example comes from the failure of inferring inhibitory and excitatory synapses from the 1986 C. elegans wiring diagram (section 1.1). This failure also challenged the significance of a structurally "complete" connectome that does not include the functional signs of synaptic 
Penultimate draft to appear in: N. Rose, T. Mahfoud, \& S. McLean (eds.). Modeling Brains: The Making and Use of Animal Models in Neuroscience and Psychiatry. Progress in Brain Research 233, Elsevier.

influence. Would a connectome that includes inhibitory and excitatory connections be "more complete", because behavioral or cognitive mechanisms could be more directly determined by this set of data than from structural information alone? One begins to sense here that the quest for completion is equally open-ended once we try to include all parameters that influence the function of neural entities. For instance, researchers that work on small invertebrate circuits have argued that the complete combination of neuromodulatory substances must be known to understand how neurons maintain stable physiological properties or perform behaviorally relevant computations (Brezina 2010, Marder 2012). In a similar vein, macroconnectomics researchers think that resting state functional connectivity studies equally contribute to the understanding of the human connectome (Sporns 2012, Glasser et al. 2016). Finally, researchers who use connectomes as technical objects typically use datasets or models instrumentally to investigate a (partially) unknown aspect of the connectome as the totality of elements and connections. So even in these cases the simultaneous reference of "connectome" is split into a technical (dataset or model) and epistemic (structure) aspect. Following Ankeny, we think that all these further experiments can revise the assumptions behind constructing connectomes. Their open-ended revisability as normative standards for assessing evidence about neural structure and its significance for understanding function is what turns connectomes into constitutively epistemic objects.

\section{Critical Neuroscience of Connectomics}

Our analysis in part one demonstrated that an understanding of connectomes as complete and permanent maps of fixed connectivity patterns is misguided. Rather than describing brain structure once and for all, connectomes reflect only transient stages in an ongoing research process. Connectomes function as revisable normative standards for further experimentation and modeling, not as a neutral and eternal depiction of brain connectivity that is independent of the research interests of neuroscientists who use them. In the second part of this chapter, we briefly probe into some of the broader implications of an understanding of connectomes as constitutively epistemic objects. This constitutive openness implies that the field of connectomics is likely to align with other disciplines of network science, technological domains such as information and 
Penultimate draft to appear in: N. Rose, T. Mahfoud, \& S. McLean (eds.). Modeling Brains: The Making and Use of Animal Models in Neuroscience and Psychiatry. Progress in Brain Research 233, Elsevier.

communication technology, and wider sociocultural domains in which networks figure prominently. But because connectomes are epistemic objects, is not settled beforehand which practices and considerations from these fields will prove productive and relevant for subsequent research. Conversely, it is likely that connectomic models of the brain will contribute to the understanding of complex systems in these other domains. In order to understand these exchanges we will now focus on some of the historical, cultural and political ramifications of network approaches to the brain. We adopt the approach of 'critical neuroscience', an interdisciplinary initiative that aims to situate the human neurosciences in their broader sociocultural ambient while revealing and debating putative misconstruals and potential ideological abuses of brain research in society (Choudhury \& Slaby 2012).

We proceed in three steps. First, we discuss the general status of models and metaphors in neuroscientific practice and show why complex real-life networks such as the Internet are indeed something like "the brain's next top model" (2.1.). Second, we take the understanding of connectomes as epistemic objects as a foundation for critiquing certain neuroscience-inspired interpretations of contemporary "network sociality" (2.2). Third, we conclude by sketching model domain realism, which strives to integrate more characteristics of real-life networks into neuroscientific modeling practices in less contentious ways (2.3).

\subsection{The brain's next top model}

Networks are suddenly everywhere: in nature, technologies, infrastructures, social life and the economy. Likewise, the brain is currently presented as an assemblage of intricate networks, reminiscent of a miniature version of the Internet. Connectomics is at the center of network thinking in the neurosciences, because it investigates how these neural networks are organized in different species. From an external perspective, it could appear as if neuroscientists are simply following a cultural trend and are uncritically projecting cultural images onto their object domain. But from an internal, history of science-informed perspective it is not surprising but rather the norm that prevailing socio-cultural or technological frameworks are applied in many different domains at once, including in advanced scientific research. In fact, this is a crucial driver of scientific work. 
Penultimate draft to appear in: N. Rose, T. Mahfoud, \& S. McLean (eds.). Modeling Brains: The Making and Use of Animal Models in Neuroscience and Psychiatry. Progress in Brain Research 233, Elsevier.

Our analysis of the "brain as network" metaphor elaborates on the studies of historian of neuroscience Cornelius Borck, who emphasizes the interaction between leading technologies, especially mechanical and communication devices, and the dominant models of brain functioning. Borck explicates the conceptual productivity of these successive historical master models as a function of their role as media:

Models in brain research typically mediated between questions of meaning, function, and significance on the one hand and the world of organic structures and mechanical functions on the other. Seen in this way, technological models are media in the multiple sense of the word, in that they transform and transmit information according to their technological specifications, and mediate between the world of biological function and the meaningful realm of day-to-day experience. They open a channel to the operations of the brain, which is structured by their technical functionality as well as by their cultural significance (Borck 2012, 118).

Each modern epoch has elaborated a certain techno-cultural framework to high sophistication, such as the steam engine, telegraphy, electricity, digital computers. Characteristically, the brain has been presented as being a paramount exemplification of the time's leading technology, so that brain function became approachable according to the model domain's operative principles. For example, the brain has been compared to a telegraphy office in the 19th century, to an electrical switch board around 1900, before being most powerfully identified with a digital computer in the mid-20th century. These technological models are generators of scientific understanding, but not in a straightforward, linear, let alone deterministic way. They are not just simplistic, time-bound projection of salient instances of technology, but contribute to the creative process of socioepistemic articulation, which is a vital part of the dynamics of scientific meaning-making in general. We use the term "articulation" to highlight that scientific practices combine discursive (i.e. representational) and non-discursive (i.e. material) elements to render a research domain intelligible (see also Rouse 2015). In the case of connectomics, researchers combine concepts and modeling assumptions (e.g., graph-theoretical topologies, network motifs, wiring rules etc.) with material elements (e.g., tracers, measurement devices, prepared brain sections) to render the domain of neural connectivity intelligible. Since researchers can creatively draw on anything as long as intelligibility is enhanced, it is no surprise that the tools for articulation can range from technology to concepts from other disciplines to socio-cultural metaphors.

Borck further holds that the incompleteness and inadequacies in the models and metaphors are not flaws, but exactly that which makes these models and metaphors so productive for research 
Penultimate draft to appear in: N. Rose, T. Mahfoud, \& S. McLean (eds.). Modeling Brains: The Making and Use of Animal Models in Neuroscience and Psychiatry. Progress in Brain Research 233, Elsevier.

in the first place. This marks an obvious link to Rheinberger's approach to epistemic objects as partly elusive zones of articulation within experimental systems. Models and metaphors likewise open up a zone for material-discursive articulation in which not everything is settled beforehand, they inspire researchers to explore lines of thought and forms of experimental practice not previously considered. Models and metaphors in scientific research can function exactly by being imperfect tools, as these imperfections become drivers of creative innovation on both conceptual and material levels.

There is, however, a flip side to this creative openness and productivity. Leading models, particularly if they are indeed culturally 'evident', might lend their target domain an inflated salience in public discourse and engender a deceptive sense of importance, for instance by letting brain connectivity appear to be the solution to all sorts of problems from mental illness to neural development to age-related degeneration. Neuroscientists today recognize that there is a hype surrounding connectomics, even though the field has yet to show whether it has the potential to lead to crucial breakthroughs in clinical and therapeutic contexts. Such hyped, conceptually productive models can also lead to the exaggeration of certain aspects of the target domain (such as: connections between regions are more relevant than the intrinsic workings of neurons or neuronal assemblies). They might also immunize researchers against counter-evidence or alternative models, as the cognitive elegance and cultural plausibility of the model might be transferred too readily from their socio-cultural domains to the target domain of research ("It must be networks."). As a consequence, the fascinating process of socio-epistemic articulation needs to be critically reflected as much as it should be promoted as a key dimension of scientific creativity.

Borck thought the Internet is not well-suited to become the leading model of the brain: "the world wide web is sometimes said to be the brain's next top model (...), and yet with its physical dispersion, the Internet is too intangible to serve as a model as the computer once did." (Borck 2012, 128). This is where we disagree. Connectome research shows how much the Internet - and other large-scale technological and social networks such as traffic patterns or logistical networkshave become a powerful modeling domain for brain research after all. Of course, connectomic researchers usually compare similarities between the connectome and real-world networks like the Internet only on an abstract level of insights and principles from graph theory. Sporns (2012), for instance, points out that space is a fundamental constraint on the topology of many physical networks, the brain and the Internet included (cf. ibid., 144). Besides such general similarities, 
however, there are three reasons why we think that the Internet has a privileged position as a model to understand the brain as a network. First, both the physical Internet and the world wide web stand in a historical continuum with previous communication technologies (e.g., telegraphy office, electrical switch boards) that served as productive metaphors for understanding the brain. Second, Internet is organized into multiple scales and layers (cf. ibid., 168), which we argue can be useful for understanding the complex topology and evolved nature of the brain, once researchers adopt model domain realism about the Internet (see section 2.3). Third, the functional similarities between the connectome and the Internet are much more specific than in the case of other networks. Both the physical Internet and the network of anatomical connections explicitly transfer information, whereas other networks primarily transfer material goods (e.g., logistic networks), or transfer information only insofar as they fulfill their primary function (e.g. maintaining ties within a social network). Furthermore, both the world wide web and the network of functional brain connections (e.g., synaptic weights) explicitly act as repositories of knowledge by storing information on websites, or implementing memory functions via Hebbian learning (Seung 2009). A recent simulation study even suggests that the physical Internet and brain circuits use the same algorithms to optimize and control the flow of data within the network (Suen and Navlakha 2017). Even though these authors admit that there is "no one-to-one mapping between mechanisms of synaptic plasticity and flow control problems" (ibid., 17), this example illustrates that that the paramount, civilization-defining real-life network called "the Internet" might be a productive model domain for understanding brain connectivity. Before exploring this productive role further in section 2.3, we want to caution against some pitfalls when the "brain as network" metaphor is uncritically aligned with contemporary forms of socio-cultural existence.

\subsection{The elusive network: Brain connectivity and contemporary network sociality}

Passages like the following one by Joseph LeDoux are unremarkable by now; after about two decades of exposure, we are prone to gloss over them without taking much notice:

Your "self," the essence of who you are, reflects patterns of interconnectivity between neurons in your brain. Connections between neurons, known as synapses, are the main channels of information flow and storage in the brain. Most of what the brain does is accomplished by synaptic transmission between neurons, and by calling upon the information encoded by past transmission across synapses (LeDoux 2002, 2). 
Network talk is seamlessly woven into the discourse of the human neurosciences, and it tends to play a critical role when knowledge about neuronal functioning is related to questions of mind, self, agency and experience, in short: where the neuronal and the mental presumably intersect. Sebastian Seung's TED talk punchline "I am my connectome" is among the most blatant statements in this regard (cf. Seung 2010, see also Seung 2012).

Not surprisingly, the notion of network has in turn figured prominently in a wide-ranging critical debate about the potentials and pitfalls of human neuroscience and its quest for establishing itself as a significant source for understanding the human subject (e.g., Martin 2000, Ehrenberg 2009, Ortega and Vidal 2011, Choudhury and Slaby 2012, De Vos and Pluth 2015). The gist of these critiques has been that neuroscientists - and their cheerleaders in the humanities ${ }^{9}$ — present accounts that (i) over-simplify the actual neuroscientific results and (ii) apply or compare the putative insights from this oversimplification to matters of culture and society. The result is a socioepistemic articulation that remains under-complex, creating the impression that the principles of brain function directly underwrite, support or even recommend specific forms of behavior, personality styles, and social organization while disfavoring other such forms. It looks as though the brain is made for a specific time-bound, often also class- or subculture-typical way of organizing and conducting a human life (Malabou 2008). The way of life in question is that of contemporary network sociality (Wittel 2001) - the highly interconnected, communicationintensive, mobile and flexible lifestyles of creative knowledge workers in the developed West, what has been analyzed by sociologists as "the new spirit of capitalism" (Boltanski and Chiapello 2005). Does that densely interconnected, highly plastic bundle of neurons under our skulls mainly support those polyvalent portfolio personalities more than other personality types (cf. Martin 2000, $582) ?$

Verbatim expressions to the effect that human brain connectivity favors networked lifestyles or forms of social organization of the developed West are rare. But once connectomics research disseminates into wider public discourse, brain and network sociality can get coarticulated in under-complex and normatively daring ways. Consider the following example. In recent years, the graph-theoretical topology of the rich club has gained prominence in connectomics (van den Heuvel and Sporns 2011, Sporns 2012, ch. 7). The rich club is technically defined as a set of high degree nodes that predominantly connect to each other and only sparsely to the rest of the network. Yet, the term "rich club" itself indicates that the concept originates from the social sphere, in which 
Penultimate draft to appear in: N. Rose, T. Mahfoud, \& S. McLean (eds.). Modeling Brains: The Making and Use of Animal Models in Neuroscience and Psychiatry. Progress in Brain Research 233, Elsevier.

individuals or groups of political and economic influence also predominantly connect and communicate with each other. Taken together, the technical and social connotations of the term inform the following passage from a university press release, in which connectomics researcher Martin van den Heuvel describes the rich club in the human brain:

The rich club $[\ldots]$ might be the G8 summit of our brain. It's a group of highly influential regions that keep each other informed and likely collaborate on issues that concern whole brain functioning. Figuring out what is discussed at this summit might be an important step in understanding how our brain works (van den Heuvel 2011).

In this example, the underlying science-the indirect nature of dMRI and macroconnectomic modeling assumptions - gets first simplified, and the resulting insights are then compared to a networked form of social organization (the G8) to highlight the importance of the brain's rich club structure. We find this socio-epistemic articulation problematic because it is made salient to the (Western) reader by reference to an organization whose membership criteria have been criticized as arbitrary and unrepresentative of the global population and economy. By comparing the (mathematically speaking) efficient rich-club topology of the brain to the G8, connectomics researchers risk to implicitly justify the G8 as an 'efficient' form of social existence in which a few powerful individuals or groups decide on issues that matter to entire societies. While network sociality stresses the interconnectedness and mobility of many individuals in contemporary societies, the socio-epistemic articulation of the rich club as G8 summit restricts the benefits of this networked lifestyle to a few members that are already well-off. We think that this risk of implicit justification is even increased when connectomes are seen as permanent descriptions of brain connectivity, with topologies such as the rich club presented as universal, species-invariant principles that transcend the specifics of human brains and culture (van den Heuvel et al. 2016).

Examples such as the above obviously require further research by science and society scholars. Yet, they illustrate how connectomics at times follows a pattern that has been often observed when (popularized) claims from the neurosciences are situated in their wider discursive, practical and organizational contexts (see Rose and Abi-Rached 2013 for an encompassing review of these contexts). In particular, Catherine Malabou has shown in an analysis of Joseph LeDoux' and Antonio Damasio's popular writings that the relationship between neuronal functioning and mental states is construed as a harmonious transition: principles of brain connectivity and neuroplasticity translate seamlessly into the image of a well-rounded and optimally adapted 
personality (cf. Malabou 2008, 65). The same tendency can be found in the public discourse surrounding functional connectivity studies of the brain 'at rest': the continuous productivity of the brain at all times harmoniously transitions into a view of the self as being always active, continually optimizing its performance at work and via productive leisure activities (Callard and Margulies 2011). The point is not to dispute that the mental is somehow materially realized in the neural substrate, but rather to challenge the particular ways in which the mind-brain relationship is spelled out by neuroscientists. As Malabou writes, "when this theoretical fissure [between mind and brain] is not recognized as such - as in the great majority of neuroscientific discourses - it runs the risk of being overwhelmed by brute, naïve ideology." (Malabou 2008, 63). This ideology—or as we prefer to say: normative imposition - is exactly the uncritical reference to the discursive template of 'network sociality' and its favored personality style, the well-connected, socially and emotionally intelligent 'network man'.

A common objection to the kind of critical analysis outlined above is that neuroscientists can always retreat to the technical definitions of connectomes or rich-club topologies instead of engaging in problematic popularizations of their research domain. In light of our preceding analyses, our reply to this objection is that the status of connectomes as constitutively epistemic objects warrants a more pro-active stance by both neuroscientists and science and society scholars. We provocatively call this the "circle of doom" argument to highlight how the connectome's ineliminable elusiveness - when not being critically reflected - turns it into an ideal plane for the projection of contentious normative proposals. First, as championed by Borck, a salient sociotechnological pattern is taken as basis for model construction in neuroscience; in this case, it is the idea of an encompassing map of all information-bearing connections in a given domain, the idea of the 'network-ome'. Second, productive brain research with this model establishes connectomes as normative standards in the field and so endows them with scientific credibility ("This is how the brain, the central organ of human mental function has been shown to be wired up"). Thirdly, connectomes can be brought forth as paradigms of organization in the human realm. Elusive and open-ended though they may be, connectomes are turned into a tool for the naturalization of social arrangements.

This maneuver of naturalization is complicated by the status of connectomes as constitutively epistemic objects. In particular, we see two potential pitfalls if the socio-epistemic articulation of the "brain as network" metaphor is not critically reflected. First, the open-ended 
Penultimate draft to appear in: N. Rose, T. Mahfoud, \& S. McLean (eds.). Modeling Brains: The Making and Use of Animal Models in Neuroscience and Psychiatry. Progress in Brain Research 233, Elsevier.

revisability of connectomes turns them into moving targets that allow the "circle of doom" to be reiterated with every new version. There is now evidence that the rich-club topology may not adequately reflect the dense connectivity of mammalian brains (Knoblauch et al. 2016). While this finding could imply that the "rich club as G8" metaphor loses its potency, the broader idea remains in place: that there is a hypercomplex, adaptive, plastic and resilient network in the brain that somehow makes us who we are. As a consequence, the "brain as network" metaphor may allow for a virtually irrefutable maneuver of naturalization in which each new rendering of brain connectivity can be (mis)used to make the seamless transition from brain architecture to mind, self and society intelligible. Second, the iterative nature of the naturalization maneuver may also allow for a pervasive and unending focus on socially accepted, socio-culturally 'normal' forms existence. By seeking but never quite reaching "normal functioning", connectome research is at risk to solidify exactly the orientation toward normalization. The standard that is always at issue but never settled may be a more powerful driver for normalization than the officially established and agreed-upon standard, the one that becomes a technical grid and backdrop of all functioning. The open-ended revisability of connectomes makes them scientifically productive for brain researchers. But that same openness could justify the search for a link between normal brain structure and function and "normal" forms of social existence as a never-ending endeavor. As pointed out in the rich club example, this risk increases when researchers do not acknowledge the constitutively epistemic character of connectomes and instead assume that there must be definite and universal wiring patterns, however hard it may be at present to discern them. The constitutively epistemic character of connectomes calls for an actively critical reflection from neuroscience and humanities scholars alike, so that undue normative impositions can be avoided. In the final section of this part we sketch how such a critical reflection on real-life networks could avoid these pitfalls and constructively inform connectomics research in less contentious ways.

\subsection{Concluding outlook: Critical neuroscience and model domain realism}

The response to these pitfalls is a call for a critical neuroscience, in the sense that modeling assumptions need to be critically reflected at each step of the way, unconstrained by the assumption of a harmonious correspondence between neural functioning and mental or social organization. Combining the lessons of Borck and Malabou, we end this chapter by sketching model domain 
Penultimate draft to appear in: N. Rose, T. Mahfoud, \& S. McLean (eds.). Modeling Brains: The Making and Use of Animal Models in Neuroscience and Psychiatry. Progress in Brain Research 233, Elsevier.

realism as a way forward for network modeling in neuroscience. Technology-inspired modeling in science tends to display a tendency towards idealization. This is to be expected, given that scientific models are supposed to be simpler and more transparent than the target system. The default assumption is that what matters about a technological arrangement is its abstract functioning principles alone - i.e. what might be captured in blue prints, diagrams or connectivity graphs. What is left out are details about material implementation, concrete socio-material contexts, historical developments - including untidy histories of tinkering and fine-tuning, such as when incompatible technological protocols have to be brought into alignment, or when unfamiliar technologies have to be geared to specific user demands. Given our considerations above, it would help to partly reverse this idealization tendency and to take more of the specificity and messiness of real-world settings into account. Of course, in order for such models to be still usable, they have to be more transparent than their target domain. But that does not entail that they must omit every bit of real-world complexity. Model domain realism therefore calls for a re-balancing of complexity and idealization in network neuroscience. This is where we return to the Internet as a potential model domain for connectomics and the human neurosciences more generally.

From the perspective of model domain realism, the most striking technical characteristic of the Internet is its combination of layers with radically different properties (Galloway and Thacker 2007, ch, 2): the application layer (user software, i.e. the "Web"), the transport layer (i.e. transmission control protocol, TCP - ensuring data arrives at its correct destination), the Internet layer (Internet Protocol, IP - the actual movement of data) and the physical layer (e.g. Ethernet the transmission technologies of the communication network). We outline this (still simplified) layer-structure here to highlight the coexistence of different topologies within the same network. For example: the Domain Name System (DNS) and the Internet Protocol (IP) are "two technologies that are intensely interconnected but are structured on radically different models of network control and organization" (Galloway and Thacker 2007, 44). The DNS is centralized in its core administration, the IP works in a fundamentally distributed manner. Applications such as digital rights management (DRM) show "how two antagonistic network topologies may work in coordination" (Galloway and Thacker 2007, 45). Such cases are by no means an exception but the rule in large-scale technological infrastructures.

At least two broader lessons might be gleaned from this exemplary model domain. First, by turning out to be much more complex and messy than expected, and thus much less optimal from 
Penultimate draft to appear in: N. Rose, T. Mahfoud, \& S. McLean (eds.). Modeling Brains: The Making and Use of Animal Models in Neuroscience and Psychiatry. Progress in Brain Research 233, Elsevier.

a functional design standpoint, the Internet might in fact match real-life human brains in key respects. It might teach us lessons in modeling less-than-ideally designed functional complexity, and in paying sufficient attention to context and historicity. The human brain presumably evolved in many successive stages, subject to many different, at times even conflicting adaptive pressures, so that a complex, multi-layered, redundant and non-ideal architecture-or even an assemblage of multiple, partly conflicting architectures — is the likely result (see also Brezina 2010, 2367). The Internet as a comparatively more realistic model domain for neural networks and function might thus help approach the brain in a more fruitful manner.

Second, turning from the natural to the social side of the story, the Internet and the world wide web in enables can function as an object lesson in studying a domain in which all relevant developments are interwoven with economic, legal, political and socio-cultural matters, sometimes giving rise to vigorous public debate, sometimes being subject to contingent developments outside the ambit of public attention. As Galloway and Thacker point out, there is no level of 'pure technicality' that could be treated in isolation from these broader strands of socio-cultural development:

[I]t is impossible to disassociate this technical topology from its motive, use, and regulation, which also make it a social topology of a different form (file-sharing communities), an economic topology with still a different form (distribution of commodities), and even a legal one (digital copyright). All of these networks coexist, and sometimes conflict with each other, as the controversy surrounding file sharing has shown. While graph theory can indeed model a number of different topologies, we prefer an approach wherein the coexistence of multiple incompatible political structures is assumed as fundamental. (Galloway \& Thacker 2007, 34)

Model domain realism proposes a similar attitude to the making and use of brain models. Instead of a smooth correspondence between a near-optimal technological design and the idea of just one basic level of social practice, researchers should embrace the entanglement of technicality and socio-political practices. If this attitude is adopted, it becomes clear why we think that both parts of this critical perspective on brain modeling belong together. The overall message of our chapter is to put the contested openness of both target domain and model domain front and center. Because of their constitutively epistemic character, what role connectomes will and should play in neuroscientific practice is an issue open to debate and contestation. Because of model domain realism, it is also an issue open to debate and contestation how the socio-political potentials of our networked plastic brains are to be unleashed, cultivated and further developed (Malabou 2008). 
This places a responsibility on neuroscientists and science and society scholars alike to inform and moderate a broader process of socio-epistemic articulation, one that is not confined to labs, scientific conferences or expert publications but extends to include members of the public, stakeholders and especially those most concerned by new developments in work, management, communication technologies, education, medicine and other focal domains of social life.

\section{References}

Ankeny, R., 2000. Fashioning descriptive models in biology: Of worms and wiring diagrams. Phil. Sci. 67, S260-272.

Ankeny, R., Chang, H., Boumans, M., Boon, M., 2011. Introduction: philosophy of science in practice. Eur. J. Phil. Sci. 1(3), 303-307.

Bargmann, C.I., Marder, E., 2013. From the connectome to brain function. Nat. Methods 10(6), 483-490.

Boltanski, L., Chiapello, E., 2005. The new spirit of capitalism. Verso, London

Brezina, V., 2010. Beyond the wiring diagram: signaling through complex neuromodulator networks. Phil. Trans. Roy. Soc. B 365(1551), 2363-2374.

Callard, F., Margulies, D.S., 2011. The subject at rest: novel conceptualizations of self and brain from cognitive neuroscience's study of the "resting state." Subjectivity 4(3), 227-257.

Catani, M., Thiebaut de Schotten, M., Slater, D., Dell'Acqua, F., 2013. Connectomic approaches before the connectome. Neuroimage 80, 2-13.

Choudhury, S., Slaby, J. (Eds.), 2012. Critical neuroscience: A handbook of the social and cultural contexts of neuroscience. Wiley-Blackwell, London.

Craver, C., 2008. Physical law and mechanistic explanation in the Hodgkin and Huxley model of the action potential. Phil. Sci. 75(5), 1022-1033.

Craddock R.C., Jbabdi S., Yan C.G., Vogelstein, J.T., Castellanos, F.X., Di Martino, A., Kelly, C., Heberlein, K., Colcombe, S., Milham, M.P. Imaging human connectomes at the macroscale. Nat. Meth. 10(6), 524-539.

De Vos, J., Pluth, E. (Eds.), Neuroscience and critique. Exploring the limits of the neurological turn. Routledge, London.

Ehrenberg, A., 2009. The weariness of the self: Diagnosing the history of depression in the contemporary age. McGill-Queens University Press, Montreal.

Fields, R.D., Woo, D.H., Basser, P.J., 2015. Glial regulation of the neural connectome through local and long-distant communication. Neuron 86(2), 374-386. 
Feest, U., 2011. What exactly is stabilized when phenomena are stabilized? Synthese 182(1), 5171.

Galloway, A.R., Thacker, E., 2007. The exploit. A theory of networks. University of Minnesota Press, Minneapolis.

Glasser M.F., Coalson T.S., Robinson E.C., Hacker C.D., Harwell J., Yacoub E., Ugurbil K., Andersson J., Beckmann C.F., Jenkinson M., Smith S.M., Van Essen D.C., 2016. A multimodal parcellation of the human cerebral cortex. Nature 536(7615), 171-178.

Goulas A., Uylings H.B., Hilgetag C.C., 2016. Principles of ipsilateral and contralateral corticocortical connectivity in the mouse. Brain Struct. Funct. Epub Aug 6.

Hammer, S., Monavarfeshani, A., Lemon, T., Su, J., Fox, M.A., 2015. Multiple retinal axons converge onto relay cells in the adult mouse thalamus. Cell Rep. 12(10), 1575-1583.

Kasthuri, N., Hayworth, K.J., Berger, D.R., Schalek, R.L., Conchello, J.A., Knowles-Barley, S., Lee, D., Vázquez-Reina, A., Kaynig, V., Jones, T.R., Roberts, M., Morgan, J.L., Tapia, J.C., Seung, H.S., Roncal, W.G., Vogelstein J.T., Burns R., Sussman D.L., Priebe C.E., Pfister H., Lichtman J.W., 2015. Saturated reconstruction of a volume of neocortex. Cell 162(3), 648361.

Levy, A., 2013. What was Hodgkin and Huxley's achievement? Br. J. Phil. Sci. 65(3), 469-492.

LeDoux, J., 2002. Synaptic self: How our brains become who we are. Penguin, New York.

Leonelli, S. and Ankeny, R., 2011. What's so special about model organisms? Stud. Hist. Phil. Sci 42(2), 313-323.

Lichtman J.W., Sanes J.R., 2008. Ome sweet ome: what can the genome tell us about the connectome? Curr. Opin. Neurobiol. 18(3), 346-353.

Livet. J., Weissman, T.A., Kang, H., Draft, R.W., Lu J., Bennis R.A., Sanes J.R., Lichtman J.W. Transgenic strategies for combinatorial expression of fluorescent proteins in the nervous system. Nature 450(7166), 56-62.

Lu, J., Tapia, J.C., White, O.L., Lichtman, J.W., 2009. The interscutularis muscle connectome. PLoS Biol. 7(2), e32.

Knoblauch, K., Ercsey-Ravasz, M. Kennedy, H. Toroczkai, Z. (2016). The Brain in Space. In Kennedy, H., Van Essen, D.C., Christen, E. (Eds.). Micro- Meso- Macroconnectomics of the Brain, Springer, Berlin, pp. 45-74.

Malabou, C., 2008. What should we do with our brain? Fordham University Press, New York.

Marder, E., 2012. Neuromodulation of neuronal circuits: back to the future. Neuron 76(1), 1-11.

Markert, S.M., Britz, S., Proppert, S., Lang, M., Witvliet, D., Mulcahy, B., Sauer, M. Zhen, M., Bessereau J.L., Stigloher, C., 2016. Filling the gap: adding super-resolution to array tomography for correlated ultrastructural and molecular identification of electrical synapses at the C. elegans connectome. Neurophotonics 3(4), 041802.

Martin, E., 2000. Mind-body problems. Am. Ethnol. 27(3), 569-590. 
Morgan, M., Morrison, M. (Eds.), 1999. Models as mediators. Perspectives on natural and social science. Cambridge University Press.

Nathanson, J.L., Yanagawa, Y., Obata, K., Callaway, E.M., 2009. Preferential labeling of inhibitory and excitatory cortical neurons by endogenous tropism of adeno-associated virus and lentivirus vectors. J. Neurosci. 161(2), 441-450.

Rheinberger, H.J., 1997. Towards a history of epistemic things: synthesizing proteins in the test tube. Stanford University Press, Stanford.

Rouse, J., 2015. Articulating the world. Conceptual understanding and the scientific image. University of Chicago Press, Chicago.

Oh, S.W., Harris, J.A., Ng, L., Winslow, B., Cain, N., Mihalas, S., Wang, Q., Lau, C., Kuan, L., Henry, A.M., Mortrud, M.T., Ouellette, B., Nguyen, T.N., Sorensen, S.A., Slaughterbeck, C.R., Wakeman, W., Li, Y., Feng, D., Ho, A., Nicholas, E., Hirokawa, K.E., Bohn, P., Joines, K.M., Peng, H., Hawrylycz, M.J., Phillips, J.W., Hohmann, J.G., Wohnoutka, P., Gerfen, C.R., Koch, C., Bernard, A., Dang, C., Jones, A.R., Zeng H., 2014. A mesoscale connectome of the mouse brain. Nature 508(7495), 207-214.

Ortega, F., Vidal, F. (Eds.), 2011. Neurocultures: glimpses into an expanding universe. Peter Lang Publishing, Bern.

Rose, N., Abi-Rached, J., 2013. Neuro: The new brain sciences and the management of the mind. Princeton University Press, Princeton.

Shine, J.M., Koyejok, O., Poldrack, R. (2016). Temporal metastates are associated with differential patterns of time-resolved connectivity, network topology, and attention. PNAS, Epub ahead of print: 10.1073/pnas.1604898113.

Seung, S., 2012. Connectome: how the brain's wiring makes us who we are. Houghton Miffin Harcourt, Boston.

Seung, S., 2010. I am my connectome. TED talk, July 2010, http://www.ted.com/talks/sebastian_seung.

Seung, S., 2009. Reading the book of memory: sparse sampling versus dense mapping of connectomes. Neuron 62(1), 17-29.

Slaby, J., Gallagher, S., 2015. Critical neuroscience and socially extended minds. TCS 32(1).

Slaby, J., Heilinger, J.-C., 2013. Lost in phenospace. Questioning the claims of popular neurophilosophy. Metodo - Int. Stud. Phen. Phil. 1(2), 83-100.

Slaby, J., Haueis, P., Choudhury, S., 2012. Neuroscience as applied hermeneutics: Towards a critical neuroscience of political theory, In: F. Vander Valk (ed.), Essays on Neuroscience and Political Theory: Thinking the Body Politic, Routledge, New York, pp. 50-73.

Sporns, O., 2013. The human connectome: origins and challenges. Neuroimage 80, 53-61.

Sporns, O., 2012. Discovering the human connectome. MIT Press, Cambridge.

Sporns, O., 2011. Networks of the brain. MIT Press, Cambridge. 
Sporns, O., Tononi, G. and Kötter S., 2005. The human connectome: A structural description of the human brain. PLoS Comput Biol 1(4), e42.

Suen J.Y. and Navlakha S., 2017. Using inspiration from synaptic plasticity rules to optimize traffic flow in distributed engineered networks. Neural Comput., 1-25. doi: 10.1162/NECO_a_00945. [Epub ahead of print].

Varshney, L.R., Chen, B.L., Paniagua, E., Hall, D.H., Chklovskii, D.B., 2011. Structural properties of the Caenorhabditis elegans neuronal network. PLoS Comput. Biol. 7(2), e1001066.

Van Essen, D.C., Smith, S.M., Barch, D.M., Behrens, T.E., Yacoub, E., Ugurbil, K., 2013a. The WU-Minn Human Connectome Project: an overview. Neuroimage 80, 62-79.

Van Essen, D.C., Jbabdi, S., Sotiropoulos, S.N., Chen, C., Dikranian, K., Coalson, T., Harwell, J., Behrens, T.E.J., Glasser, M.F., 2013b. Mapping Connections in Humans and Non-Human Primates. Aspirations and Challenges for Diffusion Imaging. In Johansen-Berg, H., Behrens, T.E.J. (Eds.), Diffusion MRI. From quantitative measurement to in vivo neuroanatomy. second ed., Academic Press, San Diego.

Van den Heuvel, M., Bullmore, E.T., Sporns, O., 2016. Comparative connectomics. Trends Cog. Sci. 20(5), 345-361

Van den Heuvel, M., Sporns, O., 2011. Rich-club organization of the human brain. J. Neurosci. 31(44), 15775-1586.

Van den Heuvel, M., 2011. Study: A rich club of the human brain. Online Press Release Indiana University Bloomington Nov 1 2011, online available at http://newsinfo.iu.edu/newsarchive/20145.html. Last accessed Aug 292016.

White, J.G., Southgate, E., Thomson, J. N., Brenner S., 1986. The structure of the nervous system of the nematode Caenorhabditis elegans. Phil. Trans. Roy. Soc. B 314(1165), 1-340.

Wittel, A., 2001. Towards a network sociality. TCS 18(6), 51-76.

Yook, C., Druckmann, S., Kim, J., 2013 Mapping mammalian synaptic connectivity. Cell Mol. Life Sci. 70(24), 4747-4757.

\section{Endnotes}

\footnotetext{
${ }^{1}$ For an example of the latter approach to neuroscience, see the discussion of the Hodgkin Huxley model of the action potential in Craver (2008) and Levy (2013).

${ }^{2}$ Ankeny follows the influential definition of Bogen and Woodward which describe phenomena as having "stable, repeatable characteristics which will be detectable by a variety of different procedures, which may yield quite different kinds of data" (quoted in Ankeny 2000, S268). In this sense, the wiring diagram displays the phenomenon of canonical C. elegans connectivity that can be detected and used independently of White et al.'s electromicrographic dataset. See Feest (2011) and Rouse (2015, ch. 7) for further philosophical discussion of phenomena.

${ }^{3}$ This notion of dual normativity (cf. Rouse 2015,244 ) is compatible with a broadly naturalistic approach to philosophy of science, because the normative scientific standards are empirically revisable (see below), and normativity in general may be explicable as a specific form of evolutionary niche construction (see Rouse 2015, ch. 3-4).
} 
Penultimate draft to appear in: N. Rose, T. Mahfoud, \& S. McLean (eds.). Modeling Brains: The Making and Use of Animal Models in Neuroscience and Psychiatry. Progress in Brain Research 233, Elsevier.

${ }^{4}$ This excerpt is from an informal interview conducted by P.H. and is reproduced with permission from the interviewee.

${ }^{5}$ The independence of these two steps does not generalize to all aspects of the HCP, let alone macroconnectomics. At the time of writing, HCP researchers published a new map of human brain areas based on structural MRI and functional connectivity data, myelin maps and task-based fMRI activations (Glasser et al. 2016), which is also called a "parcellated connectome" (ibid., fig. 4). Although certainly a normative standard, we do not count this parcellation as a wiring diagram because it does not reveal connectivity structure per se (even though each parcel is partially defined by its functional connectivity profile).

${ }^{6}$ Because connectomes as descriptive models can serve as standards for experiments on various neural functions and behavioral phenomena, we use "target" in a broader sense than Leonelli and Ankeny (2011), who specify the target as a particular phenomenon that researchers are interested in when choosing an experimental organism.

${ }^{7}$ The Brainbow technique achieves individual coloring through a combination of different fluorescent proteins that are stochastically recombined and expressed in transgenic mice (Livet et al. 2007).

${ }^{8}$ Selective plane illumination microscopy makes it possible to optimise imaging in every plane of the section, thus removing light rays that are out of focus and reducing distortions and scattering in the microscopic sections (cf. Yook et al. 2013, 4752). To achieve accurate maps of complex connectomes, super-resolution light microscopy can be combined with other techniques that identify synapses by reconstituting green fluorescent proteins between pre- and post-synaptic terminals (cf. ibid., 4751).

${ }^{9}$ By 'cheerleaders' we mean neuro-enthusiasts in various humanities disciplines who advocate approaches such as neurophilosophy, neuroaesthetics or neuropolitics. These are often only marginally engaged with actual neuroscientific practice and instead focus on broader philosophical claims and presumed prospects for future research (for critical analysis see Slaby, Haueis and Choudhury 2012, Slaby and Heilinger 2013, Slaby and Gallagher 2015). 